\title{
Development of Quantitative HPTLC Methods for Dolutegravir, Lamivudine, and Tenofovir Disproxil Fumarate in a Combination Pharmaceutical Product Using a Model Process Published earlier for Transfer of Minilab TLC Screening Methods to HPTLC-Densitometry
}

\author{
Y. Gu, B. Zeng and J. Sherma* \\ Department of Chemistry, Lafayette College, Easton, PA, USA
}

Received: 06 Aug 2019; accepted: 12 Aug 2019

\begin{abstract}
High-performance thin-layer chromatography (HPLTC)-densitometry methods are described for the analysis of the anti(retro)virals dolutegravir (D), lamivudine (L), and tenofovir disoproxil fumarate (TDF) in a pharmaceutical tablet product. To the best of our knowledge, no previous quantitative planar chromatography method has been reported in the literature for this combination formulation. The method for $\mathrm{L}$ was transferred from a thin-layer chromatography (TLC) screening method published in the Global Pharma Health Fund (GPHF) Minilab Manual designed for identification of counterfeit and substandard drug products using a model process published earlier. D and TDF are not included in the list of drugs for which TLC screening methods are published for the Minilab, but HPTLC-densitometry procedures were developed for them using the transfer process guidelines. L was analyzed simultaneously with TDF on Merck Premium Purity silica gel $60 \mathrm{~F}$ plates using the mobile phase ethyl acetatemethanol-acetone-concentrated ammonium hydroxide (30:7:3:1) and densitometric scanning at $254 \mathrm{~nm}$. D was analyzed on a second plate by scanning at $366 \mathrm{~nm}$ after chromatography with the chloroform-methanol-formic acid (32:8:2) mobile phase. Data for all three drugs are shown to meet the requirements of the model transfer process for calibration curve $r$ values, assay of tablets relative to their label values, peak purity/peak identity tests, and validation by standard addition analysis of samples spiked at $50 \%, 100 \%$, and $150 \%$ of the label value of active ingredients. A TLC screening method for TDF in the combination product was developed and published online with open access.
\end{abstract}

Keywords: dolutegravir, lamivudine, tenofovir disoproxil fumarate, TLC, HPTLC, densitometry

\section{Introduction}

A model process was previously developed [1-3] for transferring visual, semiquantitative thin-layer chromatography (TLC) screening methods for pharmaceutical products with quality defects in the Global Pharma Health Fund E.V. (GPHF) Minilab manual, Volume II [4], to quantitative high-performance TLC (HPTLC)-densitometry methods. Applications of this model process to a variety of counterfeit products were described in a recent review of advances in TLC analyses of counterfeit drugs [5]. In this paper, the process was applied to the transfer of a TLC method for the antiviral drug lamivudine (L; CAS No. 134678-17-4) in the Minilab (Volume II, 2006, Method 6.21, pp. 112-115) and for the development of HPTLC-densitometry methods for coformulated antiviral drugs dolutegravir (D; CAS No. 1051375-16-6) and tenofovir disoproxil fumarate (TDF; CAS No. 202138-50-9), both of which are not covered in the Minilab manual, in a combination product. L and TDF are simultaneously analyzed on one plate by scanning their separated fluorescence quenching bands at $254 \mathrm{~nm}$, and D on a second plate by scanning absorption at $366 \mathrm{~nm}$.

\section{Experimental}

Standard and Sample Preparation. Standard and sample solution preparation was carried out as described in the model

\footnotetext{
*Author for correspondence: shermaj@lafayette.edu.
}

process [1-3], unless otherwise specified. Standards and tablets ground using mortar and pestle were dissolved in methanol with the assistance of $10 \mathrm{~min}$ of magnetic stirring, followed by $10 \mathrm{~min}$ of sonication. Sample stock solutions were syringe-filtered to remove undissolved excipients before further dilution or direct application. Dilutions were made using appropriate volumetric flasks, volumetric pipets, and measuring pipets if necessary. Solutions were stored in Parafilm-sealed vials in a refrigerator. The sources of the sample and procedures for standard and sample solution preparation are shown in Table 1.

HPTLC. Premium purity silica gel $60 \mathrm{~F}_{254}$ plates $(20 \mathrm{~cm}$ $\times 10 \mathrm{~cm}$; Merck KGaA, Darmstadt, Germany; catalog no. 1.05648.0001) were used as received without prewashing. Calibration curves were created by spotting 7.00, 9.00, 11.0, and $13.0 \mu \mathrm{L}$ of the $100 \%$ sample solution, representing 70 $130 \%$ of the label value of the active pharmaceutical ingredient. Assays were carried out by applying $10.0 \mu \mathrm{L}$ of each sample solution in triplicate. A CAMAG (Wilmington, NC, USA) Linomat 4 was used for semiautomated bandwise standard and sample solution zone application. An application rate of $4 \mathrm{~s} / \mu \mathrm{Lwas}$ used for all solutions. The band length was $6 \mathrm{~mm}$, table speed $10 \mathrm{~mm} / \mathrm{s}$, distance between bands $4 \mathrm{~mm}$, distance from the left edge of the plate $17 \mathrm{~mm}$, and distance from the bottom of the plate $1 \mathrm{~cm}$. Mobile phases used to develop plates in a mobile-phase-vapor-saturated CAMAG twin trough chamber were ethyl acetate-methanol-acetone-

This is an open-access article distributed under the terms of the Creative Commons Attribution-NonCommercial 4.0 International License (https://creativecommons.org/licenses/by-nc/4.0/), which permits unrestricted use, distribution, and reproduction in any medium for non-commercial purposes, provided the original author and source are credited, a link to the CC License is provided, and changes - if any - are indicated. 
Table 1. Preparation of $100 \%$ standard and $100 \%$ sample solutions

\begin{tabular}{|c|c|c|}
\hline Pharmaceutical product & $100 \%$ standard solution & $100 \%$ sample solution ${ }^{a}$ \\
\hline $\begin{array}{l}\text { Dolutegravir + lamivudine }+ \\
\text { tenofovir disoproxil fumarate } \\
\left(50.0 \mathrm{mg}^{b}+300 \mathrm{mg}+300 \mathrm{mg}\right. \\
\text { Aurobindo Pharma Ltd., }\end{array}$ & $\begin{array}{l}2.00 \mu \mathrm{g} \text { per } 10.0 \mu \mathrm{L} \text { dolutegravir: dissolve } 30 \mathrm{mg} \\
\text { standard (Advanced ChemBlocks Inc., Burlingame, } \\
\text { CA, USA, Cat. ID 10313) in } 100 \mathrm{~mL} \text { methanol, } \\
\text { then dilute } 2.00 \mathrm{~mL} \text { with } 1.00 \mathrm{~mL} \text { methanol }\end{array}$ & $\begin{array}{c}2.00 \mu \mathrm{g} \text { per } 10.0 \mu \mathrm{L} \text { dolutegravir: dissolve } \\
\text { a tablet in } 100 \mathrm{~mL} \text { methanol, then dilute } \\
2.00 \mathrm{~mL} \text { with } 3.00 \mathrm{~mL} \text { methanol }\end{array}$ \\
\hline Jadcherla, India) & $\begin{array}{l}3.00 \mu \mathrm{g} \text { per } 10.0 \mu \mathrm{L} \text { lamivudine: dissolve } 30 \mathrm{mg} \\
\text { standard (Sigma-Aldrich, St. Louis, MO, USA, } \\
\text { No. PHR1365) in } 100 \mathrm{~mL} \text { methanol } \\
3.00 \mu \mathrm{g} \text { per } 10.0 \mu \mathrm{L} \text { tenofovir disoproxil fumarate: } \\
\text { dissolve } 30 \mathrm{mg} \text { standard (Sigma-Aldrich, } \\
\text { No. PHR1957) in } 100 \mathrm{~mL} \text { methanol }\end{array}$ & $\begin{array}{l}3.00 \mu \mathrm{g} \text { per } 10.0 \mu \mathrm{L} \text { lamivudine }+ \\
3.00 \mu \mathrm{g} \text { per } 10.0 \mu \mathrm{L} \text { tenofovir disoproxil } \\
\text { fumarate: dissolve a tablet in } 100 \mathrm{~mL} \text { methanol, } \\
\text { then dilute } 1.00 \mathrm{~mL} \text { with } 9.00 \mathrm{~mL} \text { methanol }\end{array}$ \\
\hline
\end{tabular}

${ }^{a}$ Concentrations indicated for $100 \%$ sample solutions are theoretical concentrations.

${ }^{b}$ The tablet contained dolutegravir sodium equivalent to $50.0 \mathrm{mg}$ dolutegravir free acid.

concentrated ammonium hydroxide (30:7:3:1) for simultaneous analysis of $\mathrm{L}\left(R_{\mathrm{F}}=0.22\right)$ and $\mathrm{TDF}\left(R_{\mathrm{F}}=0.46\right)$ on one plate and chloroform-methanol-formic acid (32:8:2) for $\mathrm{D}\left(R_{\mathrm{F}}=0.56\right)$ on another plate. Automated HPTLCdensitometry in the absorption-reflectance mode was performed using a CAMAG Scanner 3 controlled by winCATS software, with $4.00 \mathrm{~mm} \times 0.45 \mathrm{~mm}$ micro slit dimensions and a $20-\mathrm{mm} / \mathrm{s}$ scan rate. $\mathrm{L}$ and TDF quenched fluorescence of a phosphor indicator in the silica gel layer and were scanned under 254-nm ultraviolet (UV) light from a deuterium lamp, while absorbance of $\mathrm{D}$ was scanned under $366 \mathrm{~nm}$ UV light from a mercury lamp. The winCATS software created 2 calibration curves for each analysis using linear and second-order polynomial regressions of the scan areas versus applied weights of standards. Sample weights were interpolated from calibration curves based on the bracketed scan areas of the samples. Spectral comparison was used to test peak purity and identity. Validation of the developed methods was performed using standard addition by spiking at 50,100, and 150\% levels, as described by Popovic and Sherma [3].

\section{Results}

The assay results of the pharmaceutical products are displayed in Table 2, all of which were within the 85 and $115 \%$ of the label value, as specified by the United States Pharmacopeia for analysis of individual tablets. In addition, as required by the model process, the $r$ values of the calibration curves for assays and validations and those of peak purity and peak identity were at least 0.99 . The results of validation by standard addition are listed in Table 3, in which recoveries were between $95 \%$ and $105 \%$. Relative standard deviations (RSDs) for assays and validations were no greater than $3 \%$, which complies with the model process. The preferred regression mode for assays and validation of each drug was selected based on the best results in terms of higher calibration curve $r$ value, assay and validation recoveries closer to $100 \%$, and lower RSDs.

\section{Discussion}

This is the first report in the literature of a TLC procedure for determination of D, L, and TDF in a combination pharmaceutical product. The transfer of the L Minilab TLC screening method required a change in the mobile phase and applied weight. The mobile phase ethyl acetate-methanol-toluene (11:5:4) separated L and zidovudine in the Minilab method, but it did not separate D, L, and TDF, as well as the mobile phase used in our method, ethyl acetate-methanol-acetoneconcentrated ammonium hydroxide (30:7:3:1). Our mobile phase is a modified version of the mobile phase ethyl acetate-ethanol-acetone-concentrated ammonium hydroxide (4.478:0.740:0.50:0.15) used for the simultaneous estimation of L, D, and abacavir sulfate (A) in a previously published paper [6]. This latter mobile phase could not be used because the solution was not clear when the components were mixed in the proportions given in the paper. Our modified mobile phase separated D, L, and TDF and allowed the simultaneous analysis of L and TDF (Figure 1) in the combination product; $\mathrm{D}$ remained at the origin as slightly diffuse bands. A TLC screening method corresponding to the new HPTLC-densitometry method for TDF was developed and published online with open access [7].

Many mobile phases were tested for separation of $\mathrm{D}, \mathrm{T}$, and TDF, including all of those reported in the literature for silica gel TLC-densitometric analysis of products containing $\mathrm{L}+$ nevirapine $(\mathrm{N})+$ zidovudine $(\mathrm{Z}), \mathrm{L}+\mathrm{T}, \mathrm{L}+\mathrm{A}, \mathrm{L}+\mathrm{Z}+$ metronidazole $+\mathrm{N}+$ quinine, $\mathrm{L}+\mathrm{N}, \mathrm{L}+\mathrm{Z}, \mathrm{A}+\mathrm{L}+\mathrm{D}$, rilpivirine $+\mathrm{D}, \mathrm{D}$ alone, TDF alone, TDF + emtricitabine $(\mathrm{E})$, and $\mathrm{L}+\mathrm{T}+\mathrm{E}$. None of the mobile phases from these published methods or any of the others that we tested allowed

Table 2. Assay results for pharmaceutical products containing dolutegravir, lamivudine, and tenofovir disoproxil fumarate

\begin{tabular}{|c|c|c|c|c|c|c|c|}
\hline \multirow[t]{2}{*}{ Pharmaceutical product } & \multirow[t]{2}{*}{ Regression mode } & \multicolumn{2}{|c|}{ Tablet 1} & \multicolumn{2}{|c|}{ Tablet 2} & \multicolumn{2}{|c|}{ Tablet 3} \\
\hline & & Assay (\%) & $\overline{R S D}(\%)$ & Assay (\%) & $\overline{R S D}(\%)$ & Assay (\%) & $\overline{R S D}(\%)$ \\
\hline Dolutegravir & Polynomial & 91.1 & 0.901 & 101 & 1.58 & 103 & 2.43 \\
\hline Lamivudine & Linear & 86.2 & 2.65 & 86.1 & 2.69 & 88.1 & 1.57 \\
\hline Tenofovir disoproxil fumarate & Linear & 104 & 3.41 & 101 & 1.65 & 103 & 0.786 \\
\hline
\end{tabular}

Table 3. Validation results for pharmaceutical products containing dolutegravir, lamivudine, and tenofovir disoproxil fumarate

\begin{tabular}{|c|c|c|c|c|c|c|c|}
\hline \multirow[t]{2}{*}{ Pharmaceutical product } & \multirow[t]{2}{*}{ Regression mode } & \multicolumn{2}{|c|}{$50 \%$ spike } & \multicolumn{2}{|c|}{$100 \%$ spike } & \multicolumn{2}{|c|}{$150 \%$ spike } \\
\hline & & $\operatorname{Rec}^{a}(\%)$ & RSD (\%) & $\operatorname{Rec}(\%)$ & RSD (\%) & Rec (\%) & RSD (\%) \\
\hline Dolutegravir & Polynomial & 99.0 & 0.399 & 102 & 1.54 & 105 & 0.453 \\
\hline Lamivudine & Linear & 103 & 0.370 & 103 & 1.54 & 101 & 1.99 \\
\hline Tenofovir disoproxil fumarate & Linear & 102 & 0.679 & 104 & 2.54 & 104 & 1.82 \\
\hline${ }^{a}$ Rec $=$ Recovery & & & & & & & \\
\hline
\end{tabular}




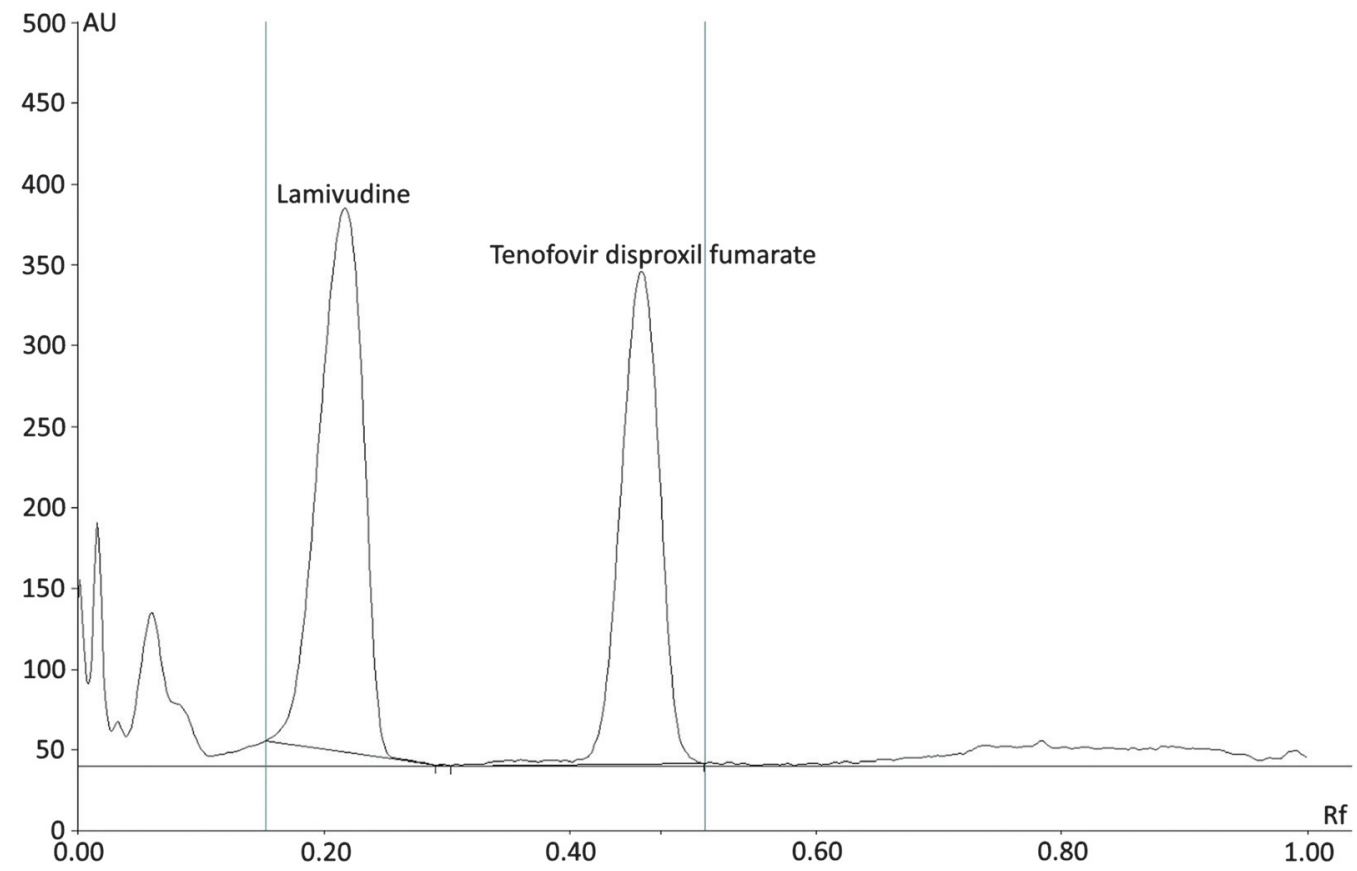

Figure 1. Densitogram of $10.0 \mu \mathrm{L}$ of dolutegravir + lamivudine + tenofovir disoproxil fumarate $100 \%$ sample solution with the mobile phase ethyl acetate-methanol-acetone-ammonia (30:7:3:1) scanned under 254-nm light, showing peaks for lamivudine $\left(R_{\mathrm{F}}=0.22\right)$ and tenofovir disoproxil fumarate $\left(R_{\mathrm{F}}=0.46\right)$

development of a simultaneous method for D + L + TDF with one sample solution and corresponding standards on one plate. Neither could we find a mobile phase that would allow analysis of $\mathrm{D}$ by a second development to move $\mathrm{D}$ off of the origin as scannable zones separated from L and TDF on the plate that was used for simultaneous analysis of $\mathrm{L}$ and TDF.

The method finally developed for analysis of D comprised a Premium Purity silica gel HPTLC plate with the mobile phase chloroform-methanol-formic acid (32:8:2), which gave a densitogram with a single peak of $\mathrm{D}$ when scanned at $366 \mathrm{~nm}$ (Figure 2). This mobile phase was a modification of the mobile phase used in a published paper to quantify $\mathrm{D}$ in bulk drug and pharmaceutical dosage form [8]. The mobile phase used in that method was methanol-chloroform-formic acid (8:2:0.5), but our modified proportions of the solvents resulted in tighter $\mathrm{D}$ zones that gave better scan peaks. The concentration of the $100 \%$ standard and sample solutions was increased compared to that in the analysis of L and TDF, because the combination product contains less $\mathrm{D}$ than $\mathrm{L}$ or TDF.

Chloroform and formic acid in our mobile phase for analysis of D are generally not allowed to be used in Minilab screening methods or in HPTLC-densitometry methods transferred by our model process. However, no mobile phase containing only Minilab-compliant components (i.e., water, methanol, ethanol, $n$-butanol, toluene, ethyl acetate, glacial acetic acid, and concentrated ammonium hydroxide) could be found to separate D from $\mathrm{L}$ and $\mathrm{T}$ in the scannable zones. The

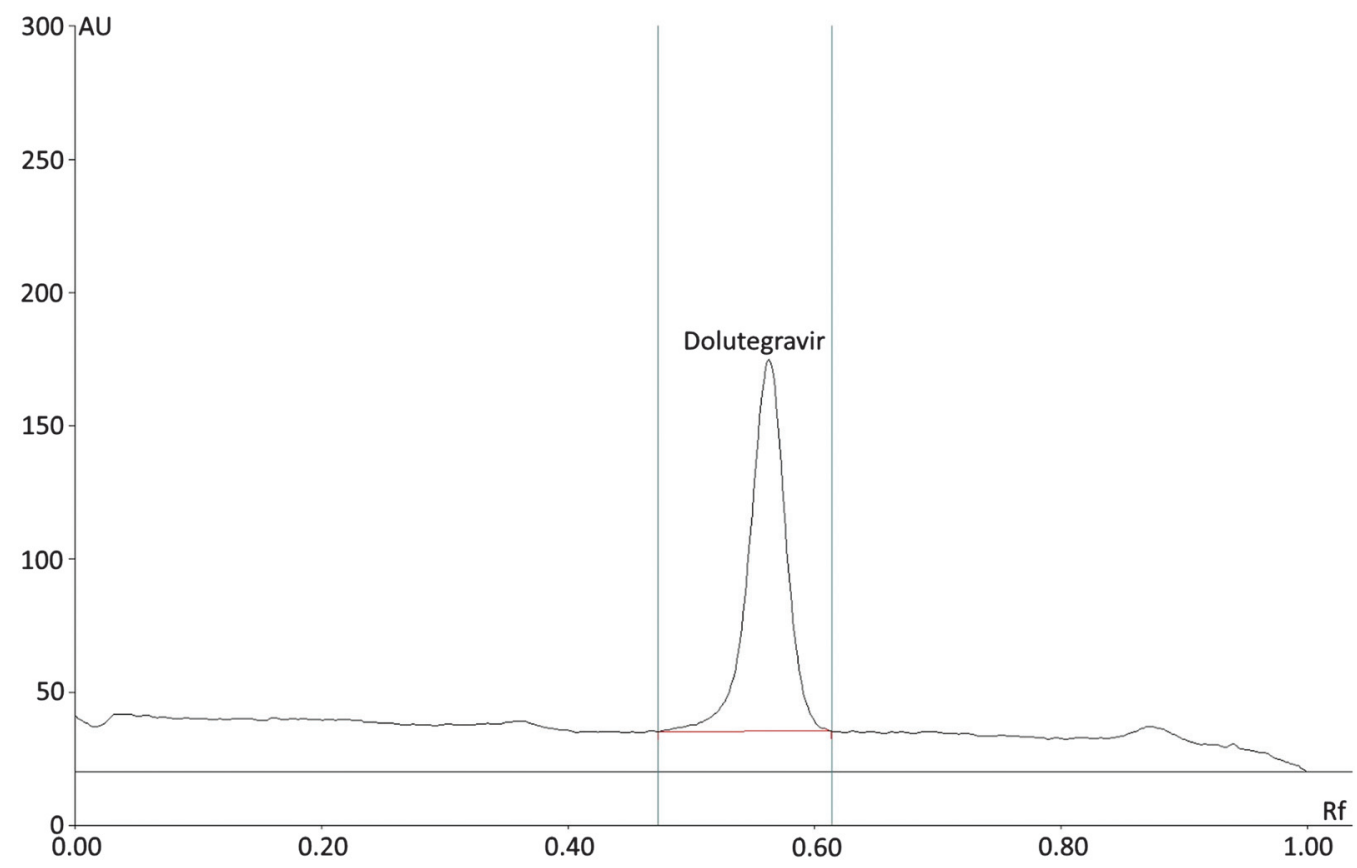

Figure 2. Densitogram of $10.0 \mu \mathrm{L}$ of dolutegravir + lamivudine + tenofovir disoproxil fumarate $100 \%$ sample solution with the mobile phase chloroform-methanol-formic acid (32:8:2) scanned under 366-nm light, showing the peak for dolutegravir $\left(R_{\mathrm{F}}=0.56\right)$ 


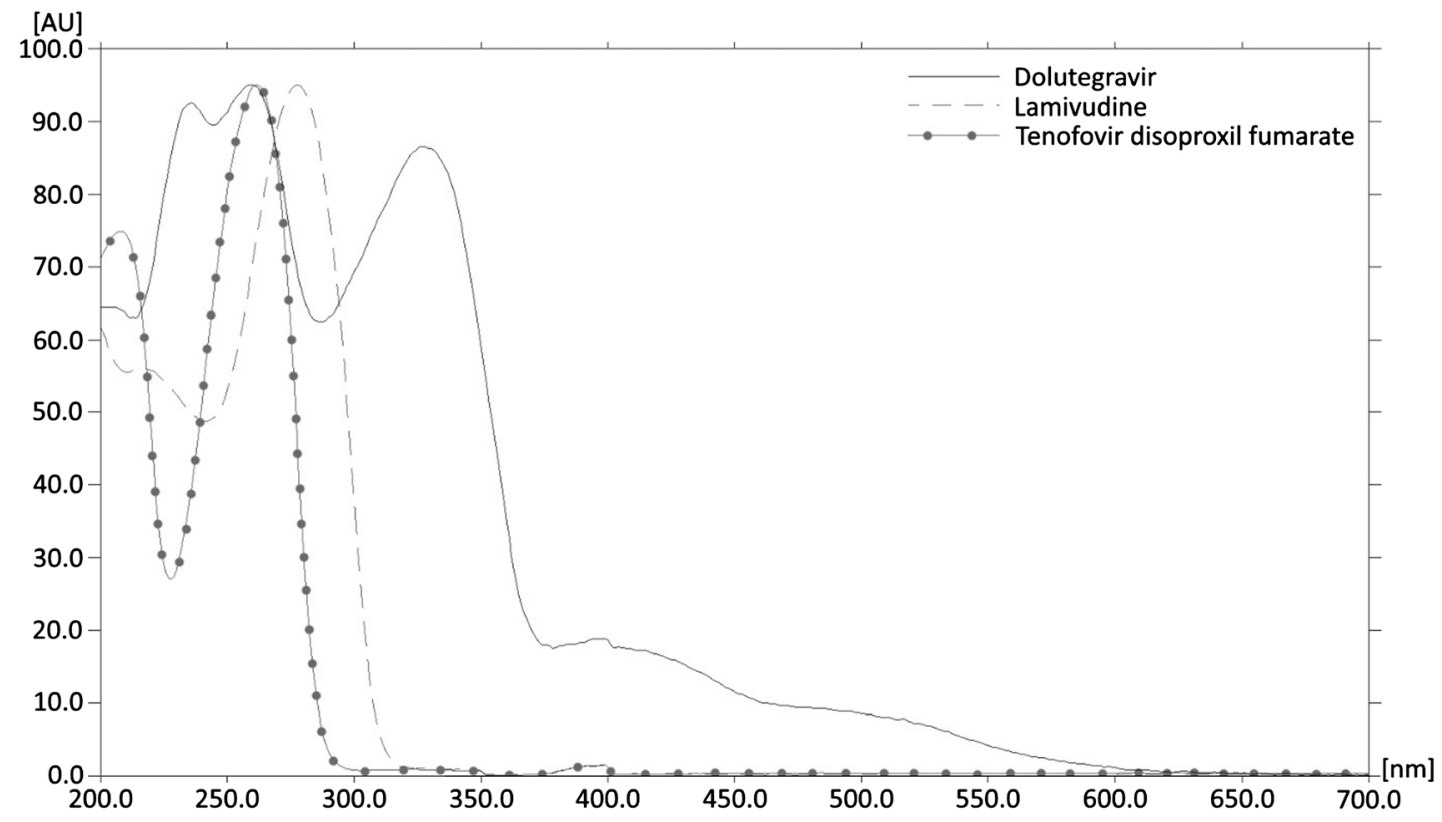

Figure 3. UV-visible absorption spectra of dolutegravir, lamivudine, and tenofovir disoproxil fumarate

development of the plate with chloroform-methanol-formic acid $(32: 8: 2)$ gave 4 bands detectable under 254-nm light: $\mathrm{L}$ at $R_{\mathrm{F}}=0.27$, TDF secondary band at 0.43 , D at 0.56 , and TDF primary band at 0.64 . TDF standard and sample solutions showed 2 bands when developed with this mobile phase, a phenomenon not observed for any other mobile phase tested. The cause of this separation into 2 bands is unknown. The D zone was not separated well enough to allow its successful densitometric analysis by scanning at $254 \mathrm{~nm}$. However, scanning in the absorption mode at $366 \mathrm{~nm}$ gave only one peak of D (Figure 2) because L and TDF did not have significant absorption at this wavelength (in situ spectra are illustrated in Figure 3). Thus, densitometric analysis of D meeting the calibration, assay, and validation guidelines of the model process was carried out at $366 \mathrm{~nm}$. Scanning in the fluorescence mode with excitation at $366 \mathrm{~nm}$ was also tested, but did not produce results as good as those in the absorption mode.

\section{Conclusion}

A previously published model process was applied to the transfer of the Minilab TLC screening method for L to a quantitative HPTLC-densitometry method and to the development of methods for quantification of T and TDF, whose screening methods are not included in the Minilab, in a three-component product. The L HPTLC-densitometry method required an increase in the applied weight and a different mobile phase that allowed a simultaneous analysis of $\mathrm{L}$ and TDF. The D analysis was performed on a second plate with a mobile phase involving 2 non-Minilab compliant components and scanning at $366 \mathrm{~nm}$.
Depending on the applications of the methods described in this paper, they should be fully validated for parameters such as accuracy, precision (repeatability and intermediate precision), specificity, linearity, range, and robustness under relevant guidelines, such as those described by the International Conference on Harmonization [9] or subjected to an interlaboratory study [10] to prove that they are suitable for their intended purpose by users.

Acknowledgments. We thank Dr. Gerd Battermann, Merck KGaA, Darmstadt, Germany, for providing the Premium Purity HPTLC plates used in our experiments. Yiru $\mathrm{Gu}$ was supported by a Camille and Henry Dreyfus Senior Scientist Mentor Program award given to Professor Sherma and also by the Lafayette College EXCEL Scholars Program. Bingsong Zeng was supported by a Professional Development Grant provided by Lafayette College given to Professor Sherma.

\section{References}

1. O'Sullivan, C.; Sherma, J. Acta Chromatogr. 2012, 24, 241-252.

2. Lianza, K.; Sherma, J. J. Liq. Chromatogr. Relat. Technol. 2013, 36, 2446-2462.

3. Popovic, N.; Sherma, J. Acta Chromatogr. 2014, 26, 615-623.

4. Global Pharma Health Fund e.V., http://www.gphf.org (accessed July 20, 2019)

5. Rabel, F.; Sherma, J. J Liq Chromatogr Mod TLC 2019, 42, 367-379.

6. Dave, K.; Sonal, D. J. Planar. Chromatogr.-Mod. TLC 2016, 39, 277-280.

7. Supplement to a Compendium of Unofficial Methods for Rapid Screening of Pharmaceuticals by Thin Layer Chromatography. http://www.layloff.net.

8. Bhaver, G. B.; Pekamwar, S.S.; Aher, K.B.; Thorat, R.S.; Chaudhari, S. R. Sci. Pharm. 2016, 84, 305-320.

9. Ferenczi-Fodor, K.; Vegh, Z.; Nagy-Tuak, A.; Renger, B.; Zeller, M. J. AOAC Int. 2001, 84, 1265-1276.

10. Kaale, E.; Risha, P.; Reich, E.; Layloff, T. P. J. AOAC Int. 2010, 93 1836-1843. 